\title{
"Relación de los factores socio-educativos y el Rendimiento Académico en los estudiantes de Agroecología y Desarrollo Rural de la Universidad Nacional San Antonio Abad del Cusco-filial Vilcabamba (Grau)"
}

\author{
Gustavo Janqui Guzmán \\ gjanqui@unamba.edu.pe \\ Facultad de Ingeniería - Escuela Académica de Ingeniería Civil. \\ Universidad Nacional Micaela Bastidas de Apurímac. \\ Mercedes Janqui Esquivel \\ Mercedes.janqui@unsaac.edu.pe \\ Facultad de Ciencias de la Salud \\ Universidad Nacional de San Antonio Abad del Cusco.
}

\section{RESUMEN}

Se determinó la situación real de los estudiantes, precisando el rendimiento académico y la relación de los factores que inciden, considerando los resultados del cuestionario y encuesta en 109 estudiantes actores de la investigación; validando que los factores tienen un valor predictivo que intervienen en el proceso aprendizaje y avance cognoscitivo. El tipo de investigación es básica y de campo; nivel de investigación analítico, descriptivo, explicativo y correlacional; método de investigación bibliográfico, analítico, deductivo, y estadístico para representar gráficamente los resultados obtenidos, a través de tabulación, análisis y como instrumento las fichas técnicas. El punto referencial y su contexto de esta problemática pueden resumirse en la identificación de las desigualdades y estados de marginación social. Los estudiantes provenientes de colegios estatales, reflejan resultados más alentadores y mejores calificaciones que de colegios privados; el uso de internet y/o biblioteca influye en el rendimiento académico por que les permite contrastar sus inquietudes y dudas; el acceso al servicio psicológico brinda en los estudiantes autoestima al sentirse sanos y aptos para el estudio; la relación entre autoridades de la universidad con los estudiantes y la infraestructura de los ambientes denotan influencia en el rendimiento. Se concluye que los resultados obtenidos, permitirán solucionar inconvenientes detectados para lograr educación de calidad.

Palabras clave: Estudiantes; factores; relación; rendimiento académico; socio educativo 


\title{
"Relationship of socio-educational factors and Academic Performance in Agroecology and Rural Development students of the San Antonio Abad National University of Cusco-Vilcabamba branch (Grau)"
}

\begin{abstract}
The real situation of the students was determined, specifying the academic performance and the relationship of the factors that influence, considering the results of the questionnaire and survey in 109 student actors of the investigation; validating that the factors have a predictive value that intervene in the learning process and cognitive advancement. The type of research is basic and field; analytical, descriptive, explanatory and correlational research level; bibliographic, analytical, deductive, and statistical research method to graphically represent the results obtained, through tabulation, analysis and as an instrument the technical sheets. The reference point and its context of this problem can be summarized in the identification of inequalities and states of social marginalization. Students from state schools show more encouraging results and better grades than from private schools; the use of the internet and / or library influences academic performance by allowing them to contrast their concerns and doubts; access to psychological service provides students with self-esteem by feeling healthy and suitable for study; the relationship between university authorities with students and the infrastructure of the environments denote influence on performance. It is concluded that the results obtained will allow to solve problems detected to achieve quality education.
\end{abstract}

Keywords: Students; factors; relationship; academic performance; educational partner

Artículo recibido: 05 de Abril 2021 Aceptado para publicación: 28 de Mayo 2021 Correspondencia: gjanqui@unamba.edu.pe Conflictos de Interés: Ninguna que declarar 


\section{INTRODUCCION}

La educación es la mejor inversión social y política que pueden hacer las familias y el Estado, pues constituye la base fundamental del desarrollo. La globalización, la excelencia y la calidad total son elementos a considerar; en consecuencia, el trabajo universitario debe orientarse a mejorar el rendimiento académico, fortalecer la formación profesional y aumentar la eficiencia y productividad. Para lograr los objetivos, es imprescindible conocer las características psicosociales e intelectuales de los estudiantes; aspectos que son determinantes e influyentes en el rendimiento académico.

De acuerdo con la Dra. Amelia Pacheco, debemos tener presente que:" Muchos de los conocimientos descubiertos en las universidades han transformado la faz del mundo y han contribuido a elevar la calidad de vida de la humanidad". Lo cierto es que la sociedad es el reflejo de la universidad; Y el medio ambiente socio familiar y económico es un laboratorio muy valioso para el desarrollo del educando dentro del claustro universitario.

Durante la labor profesional como docente en la Universidad Nacional Micaela Bastidas de Apurímac; se perciben las deficiencias de parte de los estudiantes en cuanto a logros de aprendizaje, tal fenómeno se constata y verifica previo reporte de datos concernientes a exámenes y calificaciones registradas en actas de notas, donde los estudiantes de la Escuela Profesional de Ingeniería Agroecológica y Desarrollo Rural, obtienen promedios desaprobatorios en porcentajes elevados; que es preocupación de ellos mismos, así como de las autoridades Universitarias. La labor docente y el contexto universitario, siempre es: Responder a estas problemáticas, brindando explicaciones que permitan identificar los factores determinantes del rendimiento académico en los estudiantes. Esta es una preocupación general, cualesquiera sean las poblaciones y sus realidades; porque siempre es mejorar las condiciones de vida hacia un desarrollo con equidad social, económica, cultural, política y sobre todo educativa.

La Universidad como institución formadora de profesionales y propulsora de la educación superior en el país, constituye la base fundamental del desarrollo humano y social. Sus fines y objetivos son: cultivar y desarrollar la personalidad y la inteligencia, formar a los profesionales: científicos, técnicos y directivos capaces, eficientes y creativos para que solucionen los problemas más latentes que existen en el contexto de nuestra sociedad. Así mismo extender su acción y sus servicios a la comunidad para 
promover su desarrollo integral. El punto referencial y su contexto de esta problemática pueden resumirse en la identificación de las desigualdades y estados de marginación social. Es notoria la dificultad que tienen los estudiantes, con respecto a la asimilación y acomodación de los logros de aprendizaje en su estructura cognitiva, con respecto a los temas abordados durante las sesiones de aprendizaje en clase, a pesar de la voluntad relevante de parte de ellos y de los docentes.

Existe un consenso general en cuanto a tres grandes aspectos de la educación que requieren reforma: La eficiencia de los sistemas educativos, la calidad de la educación Pública ofrecida, y, la equidad en la provisión de servicios educativos. La calidad de educación peruana es bastante baja según estándares internacionales, relacionados a aspectos básicos de lectura y escritura, así como de cálculo matemático. "La desigualdad de los resultados del aprendizaje" es un tema clave en educación, al ser la desviación estándar muy amplia. El punto referencial y su contexto de esta problemática pueden resumirse en la identificación de las desigualdades y estados de marginación social. Es notoria la dificultad que tienen los estudiantes, con respecto a la asimilación y acomodación de los logros de aprendizaje en su estructura cognitiva, con respecto a los temas abordados durante las sesiones de aprendizaje en clase, a pesar de la voluntad relevante de parte de ellos y de los docentes. La población estudiantil de esta Escuela Profesional son lugareños en un $10 \%$, mientras que los $90 \%$ restantes son procedentes de las comunidades ubicadas en sus alrededores y de otros lugares; entonces se tiene el supuesto de que esta debilidad en los estudiantes se preste a las orientaciones educativas que se produjeron en la educación básica regular; esto conlleva a decir las deficiencias en pre-requisitos que son importantes en la formación de nuevos profesionales.

La Educación Universitaria en la provincia de Cotabambas, fue implementada para cumplir un rol fundamental dentro del ámbito local y las poblaciones circundantes, así como regional y nacional; que implica que la población estudiantil, logre el avance intelectual; que es el pilar fundamental para lograr desarrollo y progreso. Sin embargo, se observa falta de interés por el avance académico de las asignaturas, deserción de estudio, desaprobación de asignaturas en cada semestre, inasistencia a las labores académicas sobre todo a las asignaturas de ciencias básicas. La deficiencia denotada de este factor conlleva a que la asimilación cognoscitiva de los temas desarrollados no sea expectante, porque no posibilitan un rendimiento eficiente. Además, los factores económico y social, 
deben ser considerados para deslindar la función y el rol que desempeñan en el desarrollo y rendimiento académico de los estudiantes.

\section{METODOS}

El tipo de investigación es básica y de campo; el nivel es analítico, descriptivo, explicativo y correlacional que permitieron determinar los factores socioeducativos que influyen en el rendimiento académico centrando su unidad de análisis en los estudiantes de la Universidad Nacional del Cusco.

Al emplear el método de investigación descriptivo, se tuvo que verificar la ubicación espacial que es la Universidad ubicada en el distrito de Vilcabamba, Provincia de Grau en el departamento de Apurímac; la ubicación temporal se realizó del 15 de Setiembre 2009 al 15 de setiembre del 2010; y para la unidad de estudio se consideró a los 109 estudiantes distribuidos según:

Tabla 1: Población estudiantil por semestres de estudios.

\begin{tabular}{|c|c|c|}
\hline Estudiantes & Cantidad & \% \\
\hline $1^{\circ}$ semestre & 25 & 22,9 \\
\hline $2^{\circ}$ semestre & 19 & 17,4 \\
\hline $3^{\circ}$ semestre & 17 & 15,6 \\
\hline $4^{\circ}$ semestre & 15 & 13,8 \\
\hline $5^{\circ}$ semestre & 14 & 12,9 \\
\hline $6^{\circ}$ semestre & 11 & 10,1 \\
\hline $7^{\circ}$ semestre & 08 & 7,4 \\
\hline total & 109 & 100.0 \\
\hline
\end{tabular}

Fuente: Elaboración propia

Población es un conjunto de todos los elementos que comparten un grupo común de características, y forman el universo para el propósito del problema de investigación (Malhotra, 2008). Considerado para delimitar la población de estudio.

Se generó el tamaño de la muestra de la población finita utilizando la ecuación matemática (1) y (2) dada por (Berenson y Levine, 1996).

$$
\begin{gathered}
n=\frac{Z^{2} p q}{e^{2}} \\
n_{0}=\frac{n N}{N+n-1}
\end{gathered}
$$


Donde:

$Z=$ Nivel de confianza $95 \%$

$e=$ error de estimación $5 \%$

$p=0.5$, Probabilidad verdadera de éxitos

$q=0.5$, Probabilidad verdadera de fracasos

$N=$ Número total de estudiantes

$n=$ tamaño de muestra

\section{Técnicas de selección de muestra:}

Se realizó un muestreó aleatorio estratificado siendo los estratos definidos por los estudiantes de los diferentes semestres académicos de la escuela profesional, compuesto por varones y mujeres. La estadística obtenida en el muestreo aleatorio estratificado nos permite inferir los parámetros de la población y generalizar los resultados para la misma.

\section{La estrategia empleada en recolección de datos fue:}

Coordinación con los miembros del Centro Federado.

La duración de la recolección de datos fue dos semanas aproximadamente.

El instrumento que se utilizó fue el formato de preguntas, el mismo que fue previamente validado antes de ser entregado a los estudiantes.

Se remarcó el carácter anónimo del formulario, así como la sinceridad de las respuestas emitidas para contribuir al éxito del estudio.

Fue preciso coordinar con los estudiantes la fecha y hora de la aplicación del instrumento, así como la clarificación de dudas que pudieran surgir. Finalmente se revisó que todos los instrumentos hayan sido respondidos en su totalidad para el control de la validez y confiabilidad. Una vez recolectados los datos, estos se sistematizaron estadísticamente para el análisis, interpretación y conclusiones finales.

A continuación, se presenta un resumen de los instrumentos utilizados en cada una de las etapas del proceso de desarrollo de la investigación, tales como la recolección de la información, el proceso de campo y el análisis de los resultados obtenidos:

- Ficha bibliográfica para el recojo de datos de los libros, revistas, artículos, etc. relacionados a la influenza de los factores socioeducativos en el rendimiento académico de los estudiantes.

- Instrumentos de medición, las notas obtenidas por los estudiantes. 
- Hojas de preguntas impresas, para aplicar las encuestas y para precisar las indagaciones durante la entrevista.

- Laptop para la elaboración de informes y procesamiento de datos

- Cámara fotográfica, para obtener imágenes relacionadas de los trabajos que se realizara.

Para la ejecución de esta investigación se analizó la problemática existente en el rendimiento académico de los estudiantes con la finalidad de encontrar los factores socioeducativos que influyen para proponer alternativas que mejoren la problemática en mención.

\section{RESULTADOS Y DISCUSIÓN}

Tabla 2: Resultados sistematizados de Rendimiento Académico

\begin{tabular}{|l|l|l|}
\hline & Frecuencia & Porcentaje \\
\hline Desaprobado & 33 & 30,3 \\
Aprobado & 76 & 69,7 \\
Total & 109 & 100,0 \\
\hline
\end{tabular}

Figura 1 Esquema de estudiantes aprobados y desaprobados

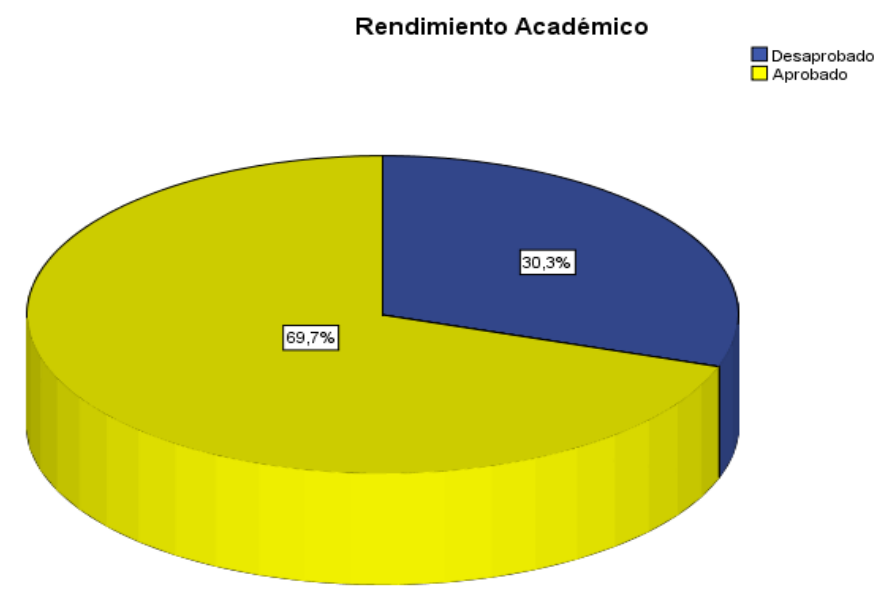


Tabla 2: Colegio donde concluyo sus estudios y el rendimiento académico

Tabla de contingencia

\begin{tabular}{|c|c|c|c|c|c|c|c|}
\hline & \multicolumn{4}{|c|}{ Rendimiento Académico } & \multirow{2}{*}{\multicolumn{2}{|c|}{ Total }} \\
\hline & & \multicolumn{2}{|c|}{ Desaprobado } & \multicolumn{2}{|c|}{ Aprobado } & & \\
\hline & & $\mathrm{N}^{\circ}$ & $\%$ & $\mathrm{~N}^{\circ}$ & $\%$ & $\mathrm{~N}^{\circ}$ & $\%$ \\
\hline \multirow{3}{*}{$\begin{array}{l}\text { En que colegió } \\
\text { concluyó sus } \\
\text { estudios } \\
\text { secundarios }\end{array}$} & Estatal & 24 & $24,5 \%$ & 74 & $75,5 \%$ & 98 & $100,0 \%$ \\
\hline & No estatal & 9 & $81,8 \%$ & 2 & $18,2 \%$ & 11 & $100,0 \%$ \\
\hline & Total & 33 & $30,3 \%$ & 76 & $69,7 \%$ & 109 & $100,0 \%$ \\
\hline
\end{tabular}

Fuente: elaboración propia

En la tabla 2, se observa que el $75,5 \%$ de estudiantes que concluyeron sus estudios secundarios en colegio estatal están aprobados, y el 24,5\% desaprueban, mientras que el $81,8 \%$ de aquellos que estudiaron en colegio no estatal están desaprobados y solo un $18,2 \%$ aprobaron.

Tabla 3: Pruebas de chi-cuadrado

\begin{tabular}{|l|c|c|c|}
\hline & Valor & gl & Sig. asintótica (bilateral) \\
\hline Chi-cuadrado de Pearson & 15,398 & 1 &, 000 \\
N de casos válidos & 109 & & \\
\hline
\end{tabular}

Se observa en la prueba de Chi-cuadrado que el valor de $\mathrm{Sig}=0,000$ es mucho menor que el nivel de significación 0,05 , por lo tanto afirmamos categóricamente que existe relación entre las variables rendimiento académico y colegio en el que culminaron sus estudios secundarios.

Figura 2. Relación variable colegio donde concluyo sus estudios secundarios, con el rendimiento académico

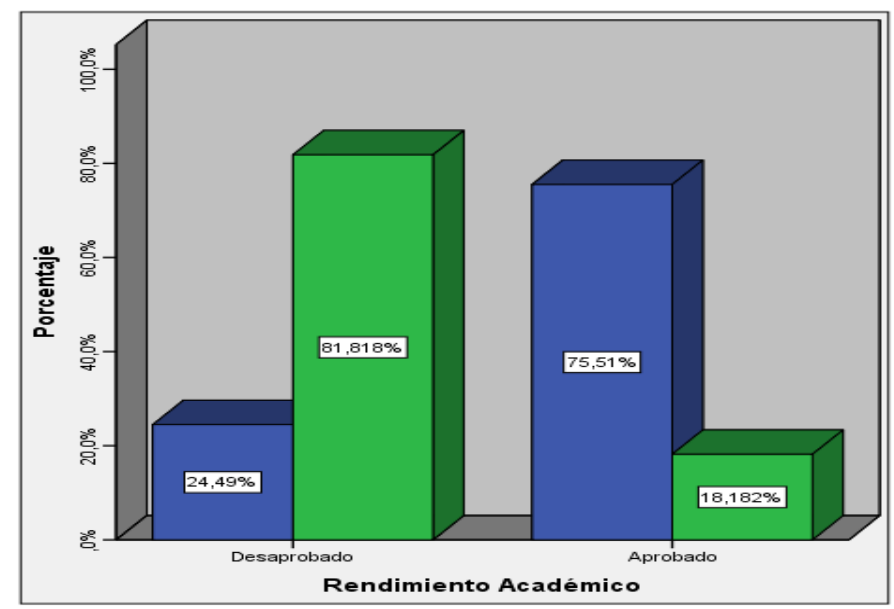

$\underset{\text { En que }}{\text { colegió }}$

concluyó
sus estudios

吕 Estatal 
Se aprecia en la Figura 2, que mejor rendimiento académico tienen aquellos que egresaron de colegios estatales porque se presume que fueron responsables en sus estudios de acuerdo a las exigencias de sus profesores; mientras que los provenientes de colegios privados desaprobaron, que se ven reflejados porque muchos de ellos son estudiantes que fueron separados de los colegios estatales por indisciplina o por rendimientos bajos.

Tabla 4: Frecuencia en el uso de internet y/o biblioteca y el rendimiento académico Tabla de contingencia

\begin{tabular}{|c|c|c|c|c|c|c|c|}
\hline & \multicolumn{4}{|c|}{ Rendimiento Académico } & \multirow{2}{*}{\multicolumn{2}{|c|}{ Total }} \\
\hline & & \multicolumn{2}{|c|}{ Desaprobado } & \multicolumn{2}{|c|}{ Aprobado } & & \\
\hline & & $\mathbf{N}^{\circ}$ & $\%$ & $\mathbf{N}^{\circ}$ & $\%$ & $\mathbf{N}^{\circ}$ & $\%$ \\
\hline \multirow{4}{*}{$\begin{array}{ll}\text { Frecuencia } & \text { uso } \\
\text { internet } & \text { y/o } \\
\text { biblioteca } & \end{array}$} & Nunca & 6 & $75,0 \%$ & 2 & $25,0 \%$ & 8 & $100,0 \%$ \\
\hline & Una vez por semana & 5 & $27,8 \%$ & 13 & $72,2 \%$ & 18 & $100,0 \%$ \\
\hline & Dos veces por semana & 16 & $23,9 \%$ & 51 & $76,1 \%$ & 67 & $100,0 \%$ \\
\hline & Todos los días & 6 & $37,5 \%$ & 10 & $62,5 \%$ & 16 & $100,0 \%$ \\
\hline Total & & 33 & $30,3 \%$ & 76 & $69,7 \%$ & 109 & $100,0 \%$ \\
\hline
\end{tabular}

Fuente: elaboración propia

En la tabla 4, se observa que aquellos que nunca frecuentan el internet y/o la biblioteca el $25,0 \%$ están aprobados y un alarmante $75 \%$ están desaprobados, mientras que los que frecuentan por lo menos una vez por semana el 72,2\% se encuentran aprobados y un $27,8 \%$ son desaprobados; mientras que el $62,5 \%$ de los que frecuentan todos los días están aprobados y un $37,5 \%$ son desaprobados.

Tabla 5: Pruebas de chi-cuadrado

\begin{tabular}{|l|c|c|c|}
\hline & Valor & gl & Sig. asintótica (bilateral) \\
\hline Chi-cuadrado de Pearson & 9,327 & 3 &, 025 \\
N de casos válidos & 109 & & \\
\hline
\end{tabular}

Se observa en la prueba de Chi-cuadrado que el valor de $\mathrm{Sig}=0,025$ es menor que el nivel de significación 0,05 por lo tanto afirmamos que existe relación entre las variables rendimiento académico y la frecuencia de uso del internet y/o la biblioteca. 
Figura 3. Relación frecuencia en el uso de internet y/o biblioteca, con el rendimiento académico
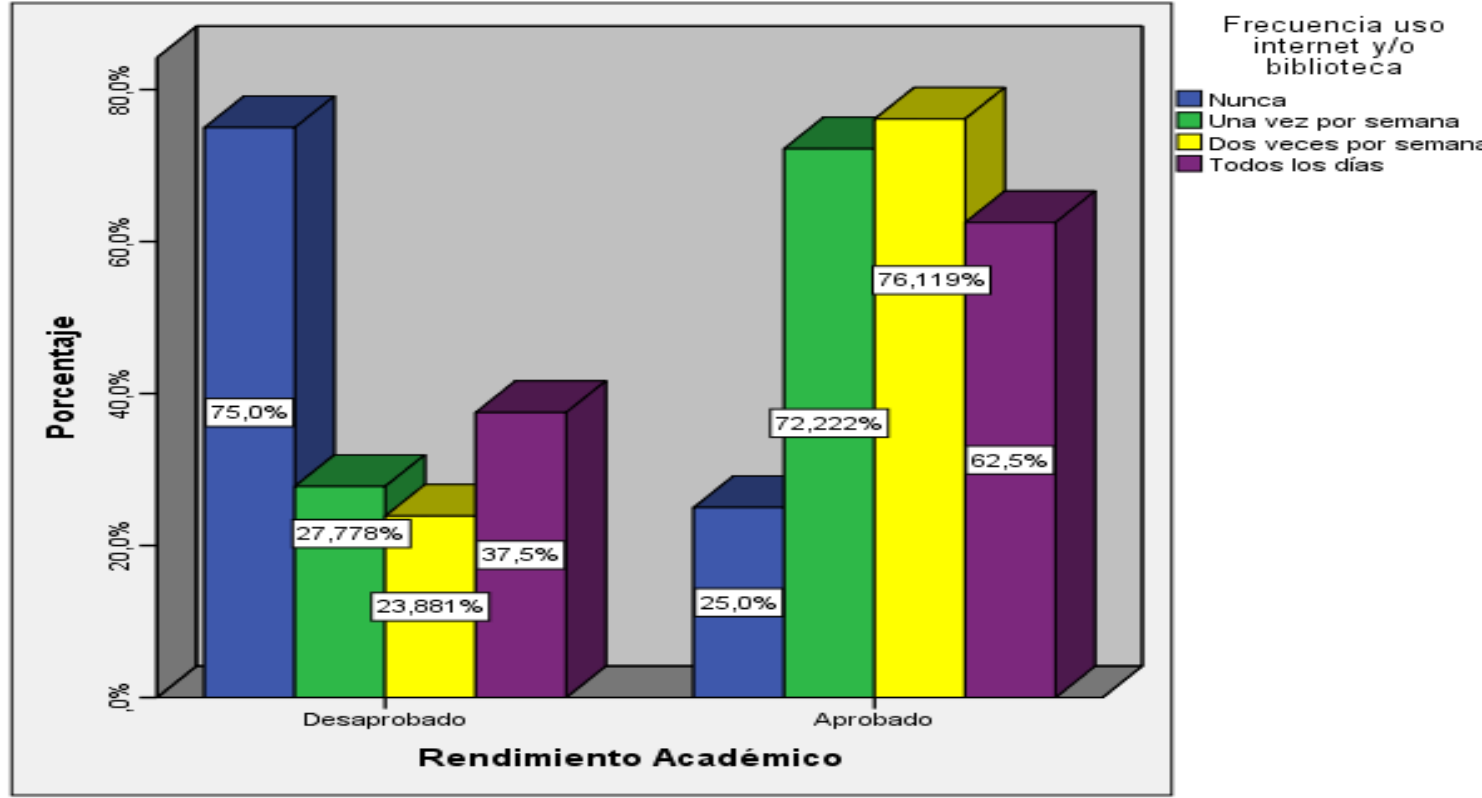

De la Figura 3, inferimos que, si los estudiantes utilizan el internet o la biblioteca para consolidar sus conocimientos, el porcentaje de aprobados incidirá notablemente sobre los desaprobados que son un porcentaje muy bajo. Por lo que podemos afirmar que es imprescindible que el estudiante utilice esta tecnología y la biblioteca para contrastar sus conocimientos y aprovechar los beneficios que brindan.

Tabla 6: Interacción estudiante autoridades y el rendimiento académico

Tabla de contingencia

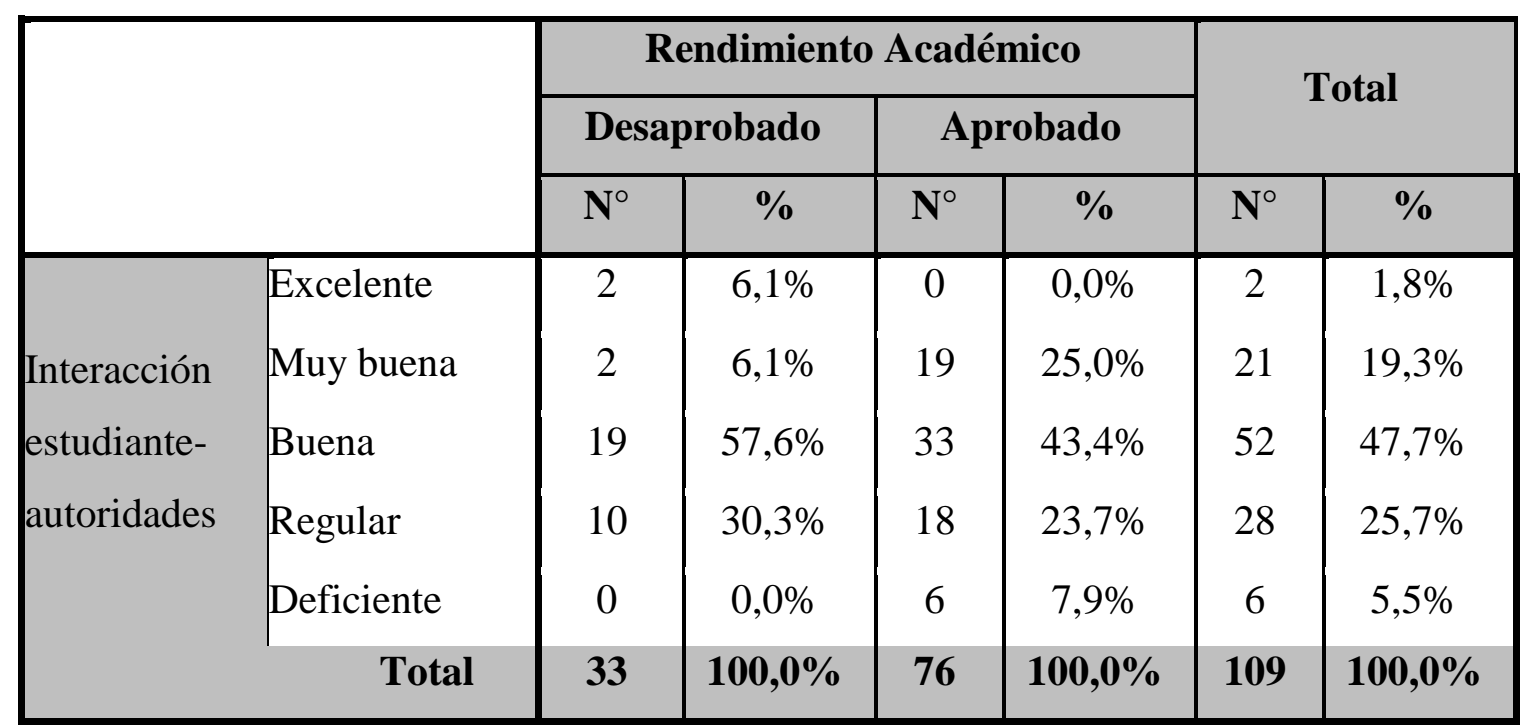

Fuente: elaboración propia 
En la Tabla 6, observamos que el 43,4\% de los estudiantes que manifiestan que su interacción con sus autoridades es buena están aprobados y el 57,6\% están desaprobados; el $25,0 \%$ de los estudiantes que aseguran que la interacción con sus autoridades es muy buena se encuentran aprobados y el 6,1\% desaprueban, el 23,7\% de los que aseguran que su interacción con sus autoridades es regular se encuentran aprobados y el 30,3\% están desaprobados; mientras que el 7,9\% de los que manifiestan que la interacción con sus autoridades es deficiente están aprobados y ningún desaprobado.

Tabla 7: Pruebas de chi-cuadrado

\begin{tabular}{|l|c|c|c|}
\hline & Valor & gl & Sig. asintótica (bilateral) \\
\hline Chi-cuadrado de Pearson & 12,854 & 4 &, 012 \\
N de casos válidos & 109 & & \\
\hline
\end{tabular}

Se observa en la prueba de Chi-cuadrado que el valor de $\mathrm{Sig}=0,012$ es menor que el nivel de significación 0,05 por lo tanto afirmamos que si existe relación entre las variables rendimiento académico y la apreciación de los estudiantes sobre la interacción existente entre ellos y sus autoridades.

\section{Figura 4. Relación Interacción estudiante autoridades con el rendimiento académico}

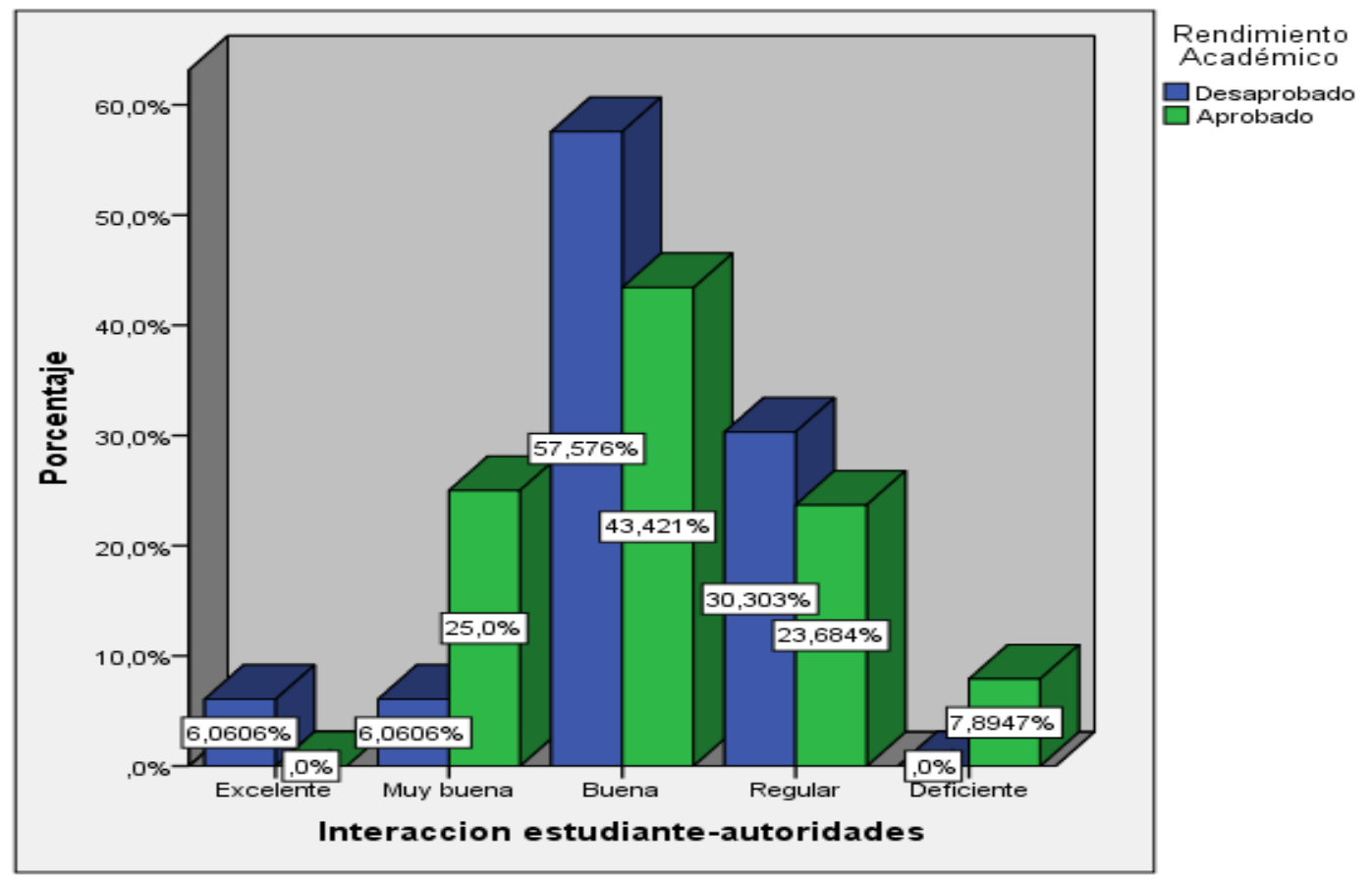


De la Figura 4, inferimos que es muy necesario que exista un intercambio fructífero y comprensión entre autoridades de la universidad con los estudiantes. Sin embargo es notorio el porcentaje de desaprobados lo que nos indica que las autoridades universitarias se aboquen a realzar el aspecto académico de los estudiantes.

Tabla 8: Infraestructura del local y el rendimiento académico

Tabla de contingencia

\begin{tabular}{|c|c|c|c|c|c|c|c|}
\hline & \multicolumn{4}{|c|}{ Rendimiento Académico } & \multirow{2}{*}{\multicolumn{2}{|c|}{ Total }} \\
\hline & & \multicolumn{2}{|c|}{ Desaprobado } & \multicolumn{2}{|c|}{ Aprobado } & & \\
\hline & & $\mathbf{N}^{\circ}$ & $\%$ & $\mathbf{N}^{\circ}$ & $\%$ & $\mathbf{N}^{\circ}$ & $\%$ \\
\hline $\mathrm{La}$ & Muy buena & 9 & $64,3 \%$ & 5 & $35,7 \%$ & 14 & $100,0 \%$ \\
\hline infraestructura & Buena & 7 & $25,9 \%$ & 20 & $74,1 \%$ & 27 & $100,0 \%$ \\
\hline de la universidad & Regular & 11 & $20,8 \%$ & 42 & $79,2 \%$ & 53 & $100,0 \%$ \\
\hline \multirow[t]{2}{*}{ es: } & Deficiente & 6 & $40,0 \%$ & 9 & $60,0 \%$ & 15 & $100,0 \%$ \\
\hline & Total & 33 & $30,3 \%$ & 76 & $69,7 \%$ & 109 & $100,0 \%$ \\
\hline
\end{tabular}

Fuente: elaboración propia

En la Tabla 8, observamos que el 79,2\% de los estudiantes que aseveran que la infraestructura de la universidad es regular están aprobados y el 20,8\% desaprobados; $74,1 \%$ de estudiantes que afirman que la infraestructura es buena son aprobados y el 25,9\% desaprobados; el 35,7\% de los que afirman que la infraestructura de la universidad es muy buena se encuentran aprobados y el 64,3\% desaprobados.

Tabla 9: Pruebas de chi-cuadrado

\begin{tabular}{|l|c|c|c|}
\hline & Valor & gl & Sig. asintótica (bilateral) \\
\hline Chi-cuadrado de Pearson & 10,861 & 3 &, 013 \\
N de casos válidos & 109 & & \\
\hline
\end{tabular}

En la Tabla 9, observamos en la prueba de Chi-cuadrado que el valor de Sig=0,013 es mucho menor que el nivel de significancia 0,05 por lo tanto afirmamos que si existe relación entre la variable rendimiento académico y la infraestructura de la universidad en la formación que actualmente reciben los estudiantes. 
Figura 5. Relación de la Infraestructura del local con el rendimiento académico
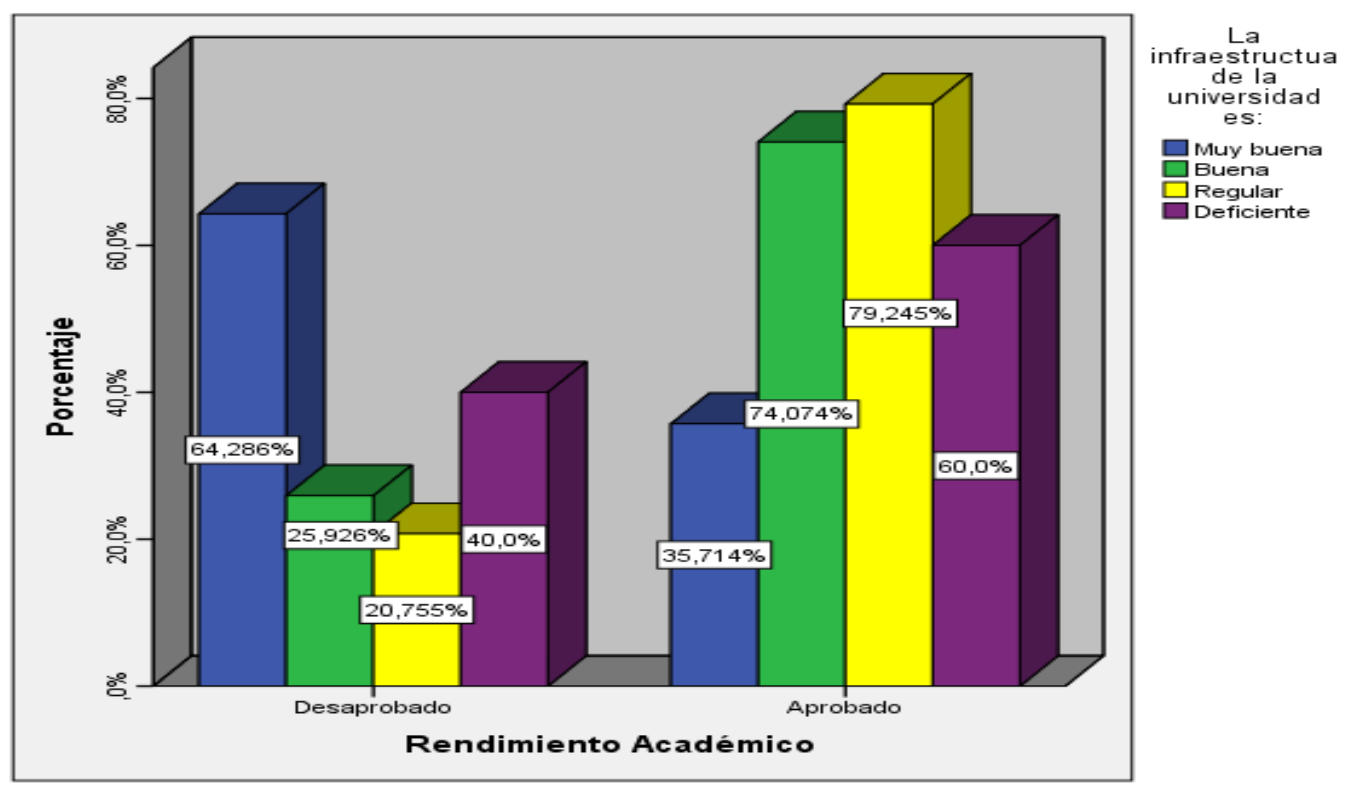

De los resultados de la Figura 5, podemos afirmar que para un buen rendimiento académico de los estudiantes se requiere que la infraestructura de la universidad debe cumplir con implementación de pizarras virtuales, amplios y ventilados salones de clase, servicios higiénicos adecuados y carpetas individuales excelentes, así como ambientes de deportes y actividades culturales.

Tabla 10: Frecuencia de acudir al servicio psicológico y el rendimiento académico

Tabla de contingencia

\begin{tabular}{|c|c|c|c|c|c|c|c|}
\hline & \multicolumn{4}{|c|}{ Rendimiento Académico } & \multirow{2}{*}{\multicolumn{2}{|c|}{ Total }} \\
\hline & & \multicolumn{2}{|c|}{ Desaprobado } & \multicolumn{2}{|c|}{ Aprobado } & & \\
\hline & & $\mathbf{N}^{\circ}$ & $\%$ & $\mathbf{N}^{\circ}$ & $\%$ & $\mathbf{N}^{\circ}$ & $\%$ \\
\hline \multirow{4}{*}{$\begin{array}{l}\text { Con que } \\
\text { frecuencia acude } \\
\text { al servicio } \\
\text { psicológico }\end{array}$} & Frecuentemente & 6 & $75,0 \%$ & 2 & $25,0 \%$ & 8 & $100,0 \%$ \\
\hline & Esporádicamente & 4 & $19,0 \%$ & 17 & $81,0 \%$ & 21 & $100,0 \%$ \\
\hline & Nunca & 23 & $28,8 \%$ & 57 & $71,2 \%$ & 80 & $100,0 \%$ \\
\hline & Total & 33 & $30,3 \%$ & 76 & $69,7 \%$ & 109 & $100,0 \%$ \\
\hline
\end{tabular}

Fuente: elaboración propia

En la Tabla 10, se observa que el $25,0 \%$ de estudiantes que afirman que acuden frecuentemente al servicio psicológico están aprobados y el $75 \%$ desaprobados; el $81 \%$ de los que visitan esporádicamente están aprobados y el 19,0\% desaprobados; el 71,2\% de los que nunca acudieron al servicio psicológico están aprobados y el 28,8\% desaprobados. 
Tabla 11: Pruebas de chi-cuadrado

\begin{tabular}{|l|c|c|c|}
\hline & Valor & gl & Sig. asintótica (bilateral) \\
\hline Chi-cuadrado de Pearson & 8,923 & 2 &, 012 \\
N de casos válidos & 109 & & \\
\hline
\end{tabular}

En la Tabla 11, se observa en la prueba de Chi-cuadrado que el valor de Sig=0,012 es significativamente menor que el nivel de significancia 0,05 por lo tanto afirmamos que si existe relación entre la variable rendimiento académico y la frecuencia con que los estudiantes acuden al servicio psicológico.

\section{Figura 6. Frecuencia de acudir al servicio psicológico con el rendimiento académico}

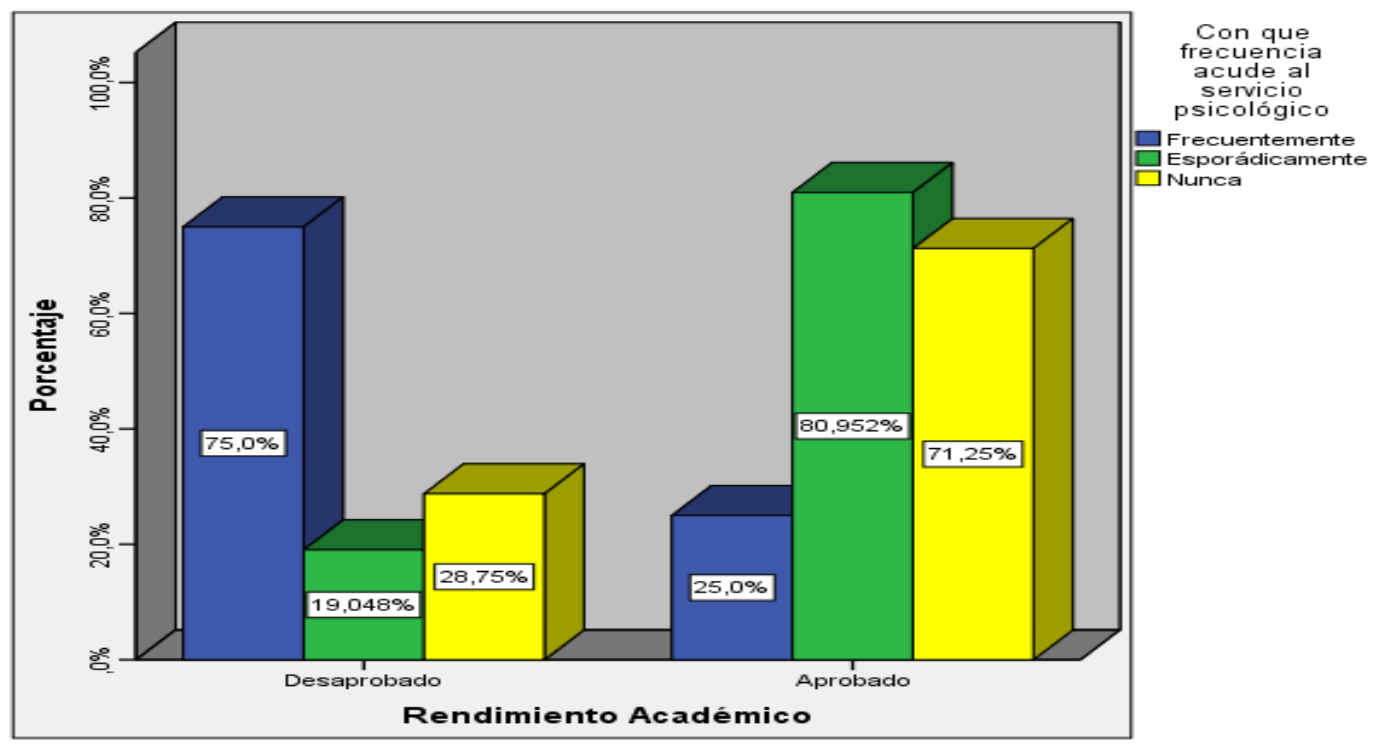

Los resultados de la Figura 6, infiere que los estudiantes para tener un buen rendimiento académico, la Universidad debe dotar el servicio permanente del profesional que constantemente acuda a los estudiantes para el diagnóstico y tratamiento efectivo

\section{CONSIDERACIONES GENERALES}

Del trabajo de investigación realizado, se distinguen las siguientes consideraciones:

Docentes que no poseen cualidades para desarrollar calidad de enseñanza para el aprendizaje. No son de especialidad por ser una nueva carrera; son contratados a tiempo parcial, no prosiguen con especializaciones, diplomados, cursos de actualización por la lejanía y falta de economía. 
Los estudiantes de la Facultad de Ingeniería Agroecológica y Desarrollo Rural de la Universidad Nacional del Cusco- Sede Vilcabamba, proceden de hogares humildes y pobres y en elevados porcentajes de hogares disfuncionales. Donde el presupuesto asignado para el estudio es mínimo; y no cubren para adquirir libros, así como no se dispone de una biblioteca especializada. Esto conlleva a que no se preparen eficientemente para rendir sus evaluaciones.

Mayor parte de los estudiantes proceden de colegios estatales y del lugar, por ende, la enseñanza aprendizaje en secundaria es bajo; denotan que los conocimientos impartidos y adquiridos en educación pre universitaria, no son suficientes ni acordes para proseguir estudios superiores; no lograron desarrollar el currículo y en muchos casos el avance curricular tuvo un avance del $60 \%$. Esto se corrobora con la falta de "base" que constituye el "cuello de botella" especialmente en las asignaturas de ciencias básicas.

La calidad global de enseñanza se aprecia en el rango de regular e Implica que el logro en asimilación cognoscitiva de los estudiantes no es eficiente porque no entienden los temas desarrollados debido a que no están preparados en las asignaturas de pre requisito.

\section{LISTA DE REFERENCIAS}

Alarcón, N. Méndez, calidad y productividad en la docencia educativa superior universitaria. Pontificia universidad católica de chile. 2003.

Auping, John. (2008). «el análisis económico de los derechos humano», primera edición, México.

Ávila Acosta, Roberto B. introducción a la metodología de la investigación - la tesis profesional, 1997.

Avila Acosta, Roberto b. Estadística Elemental. Ediciones R: A Lima, 1987.

Ary, Donald; Chesser Jacobs, Lucy y Razavieh, Asghar. Introducción a la investigación pedagógica. Nueva Editorial Interamericana S. A. México, 1982.

Centro Multinacional de Investigación Educativa (Cemie).Hernández B. Pablo. Introducción a la investigación educativa. Costa Rica, 1978.

Déniz, José, De León, Omar, (2008). «Realidades y desafíos del desarrollo económico de américa latina». Editorial Catarata. España

Flores Barboza, J. la satisfacción estudiantil, como indicador de la calidad de la educación superior. Lima, 2003. 
Lemke, Donald A. “técnicas de investigación en educación”. Editorial Perfil. UNESCO, 1977.

Lizárraga Febres, J.- Campos Tejada, S.- Benegas Esquiche, M. Estrategias de enseñanza - aprendizaje. Universidad Católica Santa María, 1996.

Muñoz, Izquierdo Carlos. (2007). «origen y consecuencias de las desigualdades educativas. Investigación realizada en américa latina». Primera Edición, México.

Vega Miranda, A. Calidad de la educación universitaria y los retos del siglo XXI ponencia presentada en el CONCAMIN, México, D.F. 2003.

Ventura, Blanco Javier; (2008). «Perspectivas económicas de la educación», ediciones universitarias de Barcelona. Edición Especial.

UNESCO. Reunión Internacional de reflexión sobre los nuevos roles de la educación superior a nivel mundial. 1991.

\section{ANEXOS}

\section{ENCUESTA REALIZADO A LOS ESTUDIANTES}

La encuesta es estrictamente confidencial, tiene por finalidad recolectar datos sobre aspectos socio económico, familiar y académicos del estudiante, a fin de disponer de un marco de referencia académica. Por tanto, agradeceremos responder con sinceridad, claridad y objetividad.

\section{Coloque un aspa(x) donde corresponde.}

\begin{tabular}{|c|c|c|}
\hline & Código o matrícula: & clo o semestre: \\
\hline \multirow[t]{2}{*}{ I } & \multicolumn{2}{|l|}{ DATOS PERSONALES } \\
\hline & \multicolumn{2}{|l|}{$\begin{array}{l}\text { 1.1 Sexo: Hombre ( ) Mujer ( ) } \\
\text { 1.2 Edad en años: } \\
\text { 1.3 Lugar de nacimiento: }\end{array}$} \\
\hline \multirow[t]{4}{*}{ II } & \multicolumn{2}{|c|}{ ANTECEDENTES EDUCATIVOS EN LA SECUNDARIA: } \\
\hline & $\begin{array}{l}2.1 \text { ¿En qué colegio terminó la educación } \\
\text { secundaria? a) Estatal ( }) \\
\text { b) No estatal ( ) }\end{array}$ & 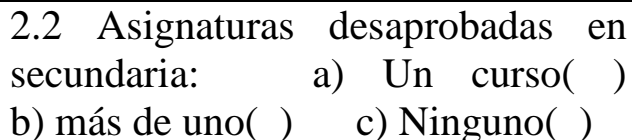 \\
\hline & $\begin{array}{l}2.3 \text { Asignaturas preferidos en secundaria: } \\
\begin{array}{ll}\text { a)Ciencias básicas( ) } & \text { b) Letras ( ) }\end{array}\end{array}$ & $\begin{array}{ll}2.4 \text { ¿Qué grado repitió en la } \\
\text { secundaria? a) } 1^{\circ}\left(\text { b) } 2^{\circ}(\text { ) c }) 3^{\circ}(\text { ) }\right. \\
\text { d) } 4^{\circ}\left(\begin{array}{lll}\text { ( ) } & \text { e } 5^{\circ}(\text { ( ) }\end{array}\right.\end{array}$ \\
\hline & $\begin{array}{l}2.5 \text { Formas de reforzar los conocimientos: } \\
\text { a) Repasando lo aprendido( ) } \\
\text { b)Haciendo resúmenes ( ) } \\
\text { c) Investigando más( ) }\end{array}$ & $\begin{array}{l}\text { 2.6 Enseñanza Aprendizaje en } \\
\text { secundaria: } \quad \text { a) bueno( ) } \\
\begin{array}{ll}\text { b) Regular ( ) } & \text { c) Malo( ) }\end{array}\end{array}$ \\
\hline \multirow[t]{2}{*}{ III } & \multicolumn{2}{|c|}{ ASPECTOS LABORALES, ECONOMICOS, SOCIAL Y CULTURAL } \\
\hline & $\begin{array}{l}3.1 \text { ¿A cuánto asciende tu presupuesto } \\
\text { mensual para estudiar?: a) } 100-149(\end{array}$ & $\begin{array}{l}3.2 \text { Vivencia familiar en el hogar: } \\
\begin{array}{lr}\text { a) Con padre y madre ( ) } & \text { b) }\end{array}\end{array}$ \\
\hline
\end{tabular}




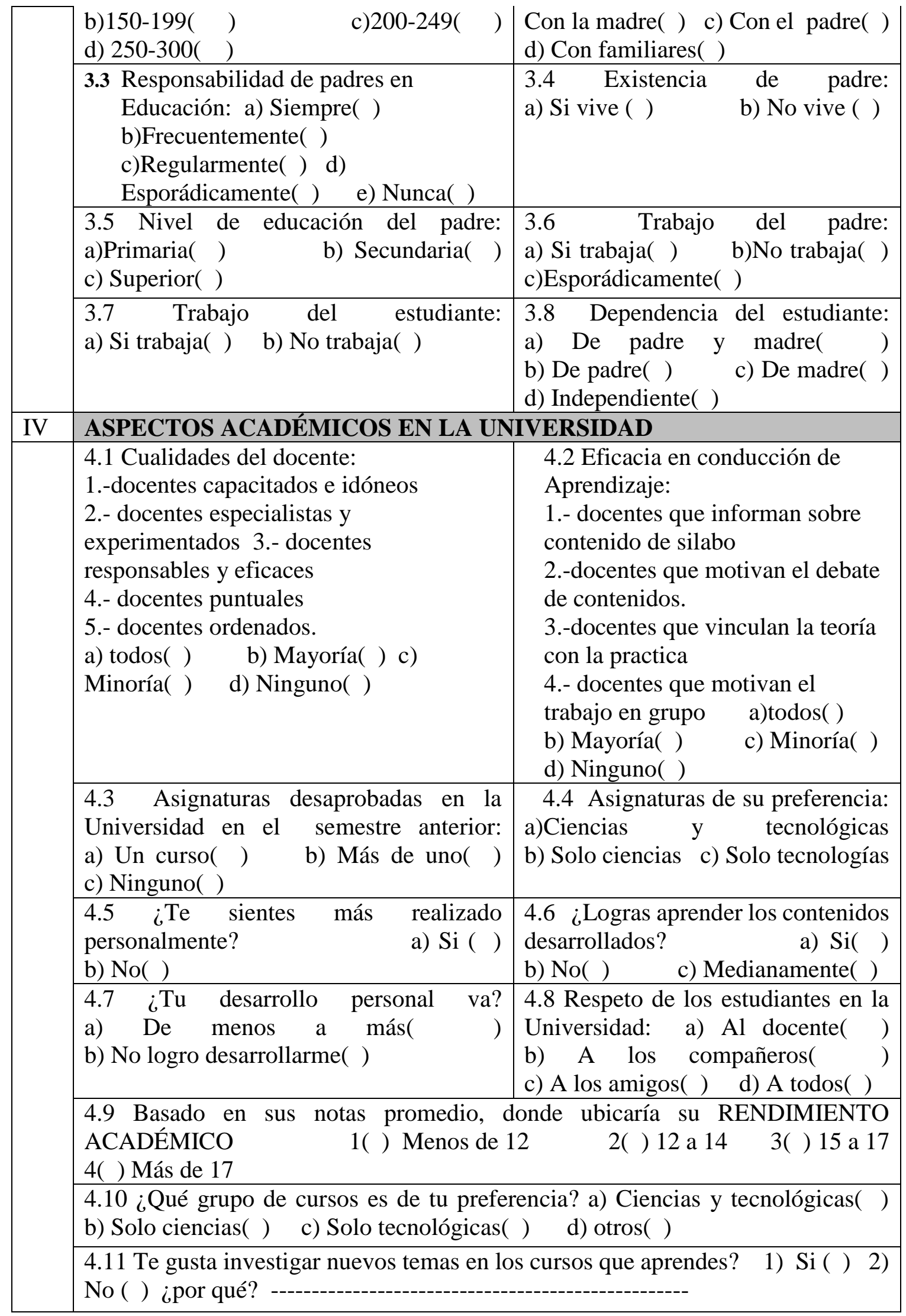




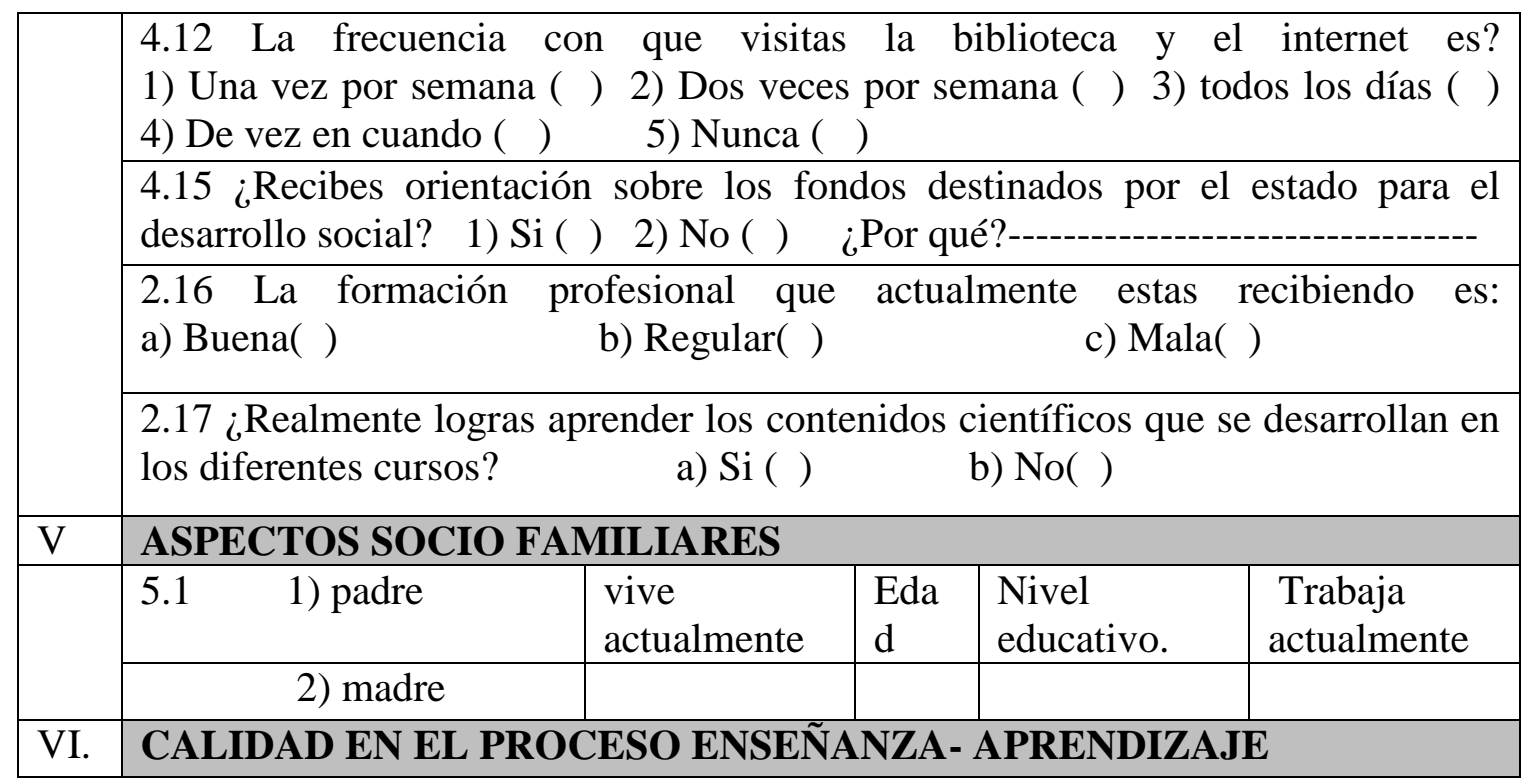

¿Cómo percibe la presencia de las siguientes cualidades en los docentes?

\begin{tabular}{|l|l|l|l|l|}
\hline \hline & Todos & Mayoría & Minoría & ninguno \\
\hline 1)Docentes capacitados e idóneos & & & & \\
\hline 2) Docentes especialistas y experimentados & & & & \\
\hline 3) Docentes responsables y eficaces & & & & \\
\hline 4) Docentes puntuales & & & & \\
\hline 5) Docentes ordenados & & & & \\
\hline
\end{tabular}

\section{¿Cómo percibe al docente en la conducción del aprendizaje?}

\begin{tabular}{|l|l|l|l|l|}
\hline & Todos & Mayoría & Minoría & ninguno \\
\hline $\begin{array}{l}\text { 1)docentes que informan sobre contenido del } \\
\text { sílabo }\end{array}$ & & & & \\
\hline $\begin{array}{l}\text { 2)docentes que motivan el debate de } \\
\text { contenidos }\end{array}$ & & & & \\
\hline $\begin{array}{l}\text { 3)docentes que vinculan la teoría con la } \\
\text { práctica }\end{array}$ & & & & \\
\hline 4)docentes que motivan el trabajo en grupo & & & & \\
\hline
\end{tabular}

\section{MB: Muy bueno B: Bueno R: Regular D: Deficiente}

\section{¿Cómo percibe a los docentes el uso de los recursos didácticos?}

\begin{tabular}{|l|l|l|l|l|l|l|}
\hline Utilización de recursos didácticos & Usa & No usa & MB & B & R & D \\
\hline Texto elaborado por el profesor & & & & & & \\
\hline Separatas seleccionadas de otros autores & & & & & & \\
\hline Bibliografía adicional & & & & & & \\
\hline Transparencias & & & & & & \\
\hline Cañón proyector & & & & & & \\
\hline Correo electrónico para consultas & & & & \\
\hline
\end{tabular}




\begin{tabular}{|l|l|l|l|l|}
\hline $\begin{array}{l}\text { ¿Cómo percibe los instrumentos de evaluación para comprobar el logro de } \\
\text { objetivos que usan los profesores? }\end{array}$ & Todos & Mayoría & Minoría & Ninguno \\
\hline Instrumento & & & & \\
\hline Fichas de control de lectura & & & & \\
\hline Ejercicios de aplicación & & & & \\
\hline Resolución de casos & & & & \\
\hline Monografías & & & & \\
\hline Pruebas de conocimientos & & & & \\
\hline Exposiciones en clase & & & & \\
\hline Otros & & & & \\
\hline
\end{tabular}

\begin{tabular}{|ll|}
\hline ¿Cómo calificaría en forma global, la calidad del proceso-enseñanza? \\
\hline 1) Excelente $\square$ & 4) Regular \\
2) Muy buena $\square$ & 5) Deficiente \\
3) Buena $\square$ & $\square$ \\
\hline
\end{tabular}

\begin{tabular}{|l|l|l|l|l|l|}
\hline ¿Cómo percibe la calidad en la interacción humana entre docentes-alumnos? \\
\hline \hline Interacción humana entre: & Excelente & M. buena & Buena & Regular & Deficiente \\
\hline 1)Estudiantes - estudiantes & & & & & \\
\hline 2) Estudiantes - docentes & & & & & \\
\hline 3) Estudiantes - autoridades & & & & & \\
\hline 4) Docente - docente & & & & & \\
\hline
\end{tabular}

\section{¿Cómo percibe la frecuencia de uso de los servicios de soporte académico?}

\begin{tabular}{|l|l|l|l|l|l|}
\hline \hline Servicios/ Frecuencia & A & B & C & D & E \\
\hline Biblioteca & & & & & \\
\hline Hemeroteca & & & & & \\
\hline Internet & & & & & \\
\hline Médico & & & & & \\
\hline Laboratorio de análisis clínicos & & & & & \\
\hline Clínica odontológica & & & & & \\
\hline Consultorio Psicológico & & & & & \\
\hline Consejería espiritual & & & & & \\
\hline
\end{tabular}

A: siempre B: Frecuentemente C: Regularmente D: Esporádicamente E: Nunca

¿Cómo percibe la calidad en su desarrollo personal?

¿Siente que su desarrollo como persona va de menos a más? 1 1) Si ( ) 2 2) No ( )

¿Se siente Ud. más realizado como persona y estudiante?

1) Sí ( ) 2) No ( )

\section{¿Cómo calificaría en forma global la calidad del ambiente académico?}

Excelente
Muy bueno
Aceptable
Regular
Deficiente


¿En cuál de estos tres rubros, cree usted que la Universidad es mejor?

1) Calidad en el proceso Enseñanza- Aprendizaje

2) Calidad en el ambiente académico

3) Calidad en la infraestructura 